\title{
Changes of Intracerebral Ventricular Width in Children with Leukemia at Diagnosis, During Treatment and in Follow-Up
}

\author{
Mergen M1, Graf N2*, Scheid S', Schubmehl H², \\ Backens $\mathbf{M}^{1}$ and Reith $\mathbf{W}^{1}$ \\ ${ }^{1}$ Saarland University Medical Center, Department for \\ Diagnostic and Interventional Neuroradiology, Homburg, \\ Germany \\ ${ }^{2}$ Saarland University Medical Center, Department of \\ Pediatric Oncology and Hematology, Homburg, Germany \\ *Corresponding author: Norbert Graf, Saarland \\ University Medical Center, Kirrberger Str. 100, 66421 \\ Homburg, Germany
}

Received: J une 14, 2021; Accepted: J uly 13, 2021; Published: July 20, 2021

\begin{abstract}
Background: Acute leukemias are the most common cancer in childhood. Due to multimodal treatment, cure rates are excellent today. Therefore, acute toxicities as well as long-term sequelae of the disease and the treatment need to be examined. Neurotoxicity is one of the most important sequelae. Nevertheless, correlation to intracerebral findings in MRI for children with leukemias is scarce. The aim of this study is to examine the changes of intracerebral ventricular width in children treated for leukemia.
\end{abstract}

Material and Methods: In this retrospective analysis, 95 patients (52 male, 43 female) with a primary diagnosis of leukemia (78 ALL, $17 \mathrm{AML}$ ) between 2007 and 2017 from a single center were included (median age at diagnosis: 5 years). We analyzed 170 cranial MRI scans (T2, FLAIR axial) and measured ventricular width as the greatest distance between the anterior ventricular horns (GDAH).

Results: GDAH enlarges significantly during follow-up in children up to 6 years, those without morphological brain atrophy and in patients with relapse or high-risk leukemia. During the first year after diagnosis male patients, those with ALL, with intermediate risk and with intracerebral pathomorphological changes were most affected. A normalization of GDAH was possible.

Conclusion: Treatment of leukemia during childhood can increase ventricular width. This may explain to some degree the known negative impact on neurocognitive functions. Based on these results and those from literature, routine MRIs at diagnosis and during follow-up in children with acute leukemias need to be discussed. Future work has to correlate these findings with neurocognitive function.

Keywords: Cerebral MRI; Childhood acute leukemias; Ventricular width; Morphological changes

\section{Ablbreviations}

ALL: Acute Lymphoblastic Leukemia; AML: Acute Myeloid Leukemia; CNS: Central Nervous System; GDAH: Greatest Distance between Anterior Ventricular Horns; MRI: Magnetic Resonance Imaging; MTX: Methotrexate; PACS: Picture Archiving and Communication System

\section{Introduction}

One third of all childhood cancers are leukemias with Acute Lymphoblastic Leukemia (ALL) being the most common ( $\approx 25 \%)$ [1]. More than $90 \%$ of children with ALL can be cured today. Even for children with AML outcome is steadily improving now exceeding an overall survival rate of $70 \%$ [1]. This success story is mainly based on multimodal therapies within prospective multicentric and randomized clinical trials. As current treatment strategies are very effective, long-term sequelae of the disease and the treatment are of major concern. Neurocognitive sequelae are well known [27]. Therefore, special attention has to be given to morphological changes of the Central Nervous System (CNS) [8-22]. However, large-scale investigations with a special focus on neuroradiological changes during and after multimodal treatment of leukemia in early childhood are sparse.

The median age at diagnosis of ALL and AML is around 4.5 years [23], still a vulnerable phase in the development of the brain, explaining why neurotoxic treatments can cause neurocognitive impairments related to morphological changes.

The aim of this study is to investigate if changes of the greatest distance between the anterior ventricular horns measured in MRI (GDAH), could be a marker for neurotoxicity [24-28], in children with acute leukemia during treatment and follow-up.

\section{Materials and Methods}

In this retrospective study, we included all children with acute leukemias diagnosed at the Department of Pediatric Oncology and Hematology at the Saarland University Medical Center in Homburg, Germany, over a 10-year period between 2007 and 2017. Included were all children who did receive cranial MRIs. MRIs were performed initially at the time of diagnosis to mainly exclude CNS involvement
Austin J Radiol - Volume 8 Issue 7 - 2021

ISSN : 2473-0637 | www.austinpublishing group.com

Graf et al. @ All rights are reserved
Citation: Mergen M, Graf N, Scheid S, Schubmehl H, Backens M and Reith W. Changes of Intracerebral Ventricular Width in Children with Leukemia at Diagnosis, During Treatment and in Follow-Up. Austin J Radiol. 2021; 8(7): 1148 
and at any time during or after treatment if neurological symptoms occurred. In addition, in children with pathological cranial MRIs (cMRI) at any time subsequent MRIs were done during follow-up. In this cohort of 96 patients, 78 were diagnosed with ALL, 17 with AML and one with biphenotypic leukemia (female). For analysis, the patient with biphenotypic leukemia, having only one MRI, was excluded. The median age at initial diagnosis, was 5 years ranging from 5 months to 22 years with 47 patients younger than 6 years. According to the classification of ALL and AML 26 patients belonged to the high-risk group, which is defined with one of the following items:

\section{ALL (Acute Lymphoblastic Leukemia)}

Prednisone poor response ( $\geq 1000 / \mu 1$ leukemic cells at day 8$)$, no cytomorphological remission at day 33 , translocation $\mathrm{t}(9 ; 22)$ respectively $\mathrm{BCR} / \mathrm{ABL}$ or $\mathrm{t}(4 ; 11)$ respectively $\mathrm{MLL} / \mathrm{AF} 4, \mathrm{MRD}$ (Minimal residual disease) $\geq 10-3$ before protocol M (ALL-BFM 2000), hypodiploid (AIEOP-BFM ALL 2009).

\section{AML (Acute Myeloid Leukemia)}

Patients showing the following cyto- or molecular genetic aberrations: $12 \mathrm{p} / \mathrm{t}(2 ; 12)$, isolated monosomia $7, \mathrm{t}(4 ; 11), \mathrm{t}(5 ; 11)$, $\mathrm{t}(6 ; 11), \mathrm{t}(6 ; 9), \mathrm{t}(7 ; 12), \mathrm{t}(9 ; 22)$, WT1mut/FLT-ITD.

At least one cranial MRI was done during treatment or follow up for every patient. $52 \%$ of the children had one MRI, $29 \%$ had two, $7 \%$ three and $12 \%$ four. Overall, 171 MRI scans could be analyzed (Supplementary Table S1). The average time of follow-up was 6.83 years.

In the first 30 days (Timepoint 1 (TP1)) after diagnosis $86 \mathrm{MRI}$ scans were performed, 44 MRIs in the period between $>30$ days and $<365$ days (Timepoint 2 (TP2)) and 40 later than 1 year (Timepoint 3 (TP3)). We have gathered the MRIs in these time periods because they represent the initial induction treatment, the reinduction and start of maintenance, followed by maintenance to the end of treatment and follow-up.

Treatment was given according to the BFM protocols for ALL (AIEOP-BFM ALL 2009 [29], ALL-BFM 2000 [30,31]) and AML (AML-BFM 2004 [32]). Besides vincristine, intrathecal and intravenous Methotrexate (MTX), and intrathecal Cytarabin were given representing potentially neurotoxic cytostatic drugs. Prophylactic cranial irradiation was applied in one patient with T-ALL, 7 others received cranial irradiation due to CNS involvement. The age range of those receiving cranial irradiation was between 2 and 19 years. 19 patients of the cohort relapsed.

We collected relevant clinical parameters including risk group, CNS- involvement, irradiation, stem cell-transplantation and site of relapse from the medical records of the patients.

Imaging studies were retrieved from the hospital's PACS system. For analysis, we used axial T2 scans and flair- sequences. In each scan, ventricular width was defined as the largest distance between the anterior ventricular horns (GDAH, Figure 1) from the left to the right outside lateral wall. In addition, we looked for brain atrophy, sinus thrombosis, white matter changes and ischemia, summarized as intracerebral pathomorphologies in general. In this study, brain atrophy was graded by an experienced neuroradiologist as a visible

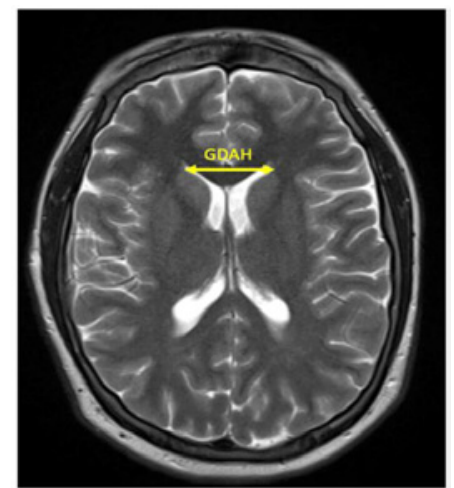

Figure 1: GDAH is defined as the largest distance between the anterior ventricular horns and shown by the arrow.

increase of the outer cerebral fluid spaces.

Anonymized clinical data and measurements from the MRI were stored in a database (Numbers for MAC, version 6.0) after linkage of clinical data and imaging studies for analysis. Privacy policy adhered to the European General Data Protection Regulation (GDPR).

Ethical approval for the clinical trials AIEOP-BFM ALL 2009, ALL-BFM 2000 and AML-BFM 2004 and accompanying research was given by the ethical committee of the Saarland Medical Association Ethics Review Board for the different leukemic trials (ID-numbers: $51 / 06,15 / 08,53 / 13,58 / 13$ ) and for quality control (ID-number: 101/11). Informed consent for the MRI scans was obtained from patients ( $>14$ years) and parents or legal guardians.

\section{Statistics}

Statistics were done using "IBM SPSS Statistics", version 25, 64bit, for Mac. Descriptive analyzes as well as comparing statistics have been made. T-tests for connected samples were used for comparison of GDAH in the same group over time and t-tests for disconnected samples were applied to compare different subgroups at the same time. $\mathrm{P}<0.05$ was defined as statistically significant $(\alpha=0.05)$. Graphic illustrations were made in form of scatter plots, boxplots, bar- and line diagrams. To verify intra- and interobserver variability of GDAH in randomly selected cranial MRIs, GDAH was measured by two independent observers in 10 different MRIs, each for 3 readings by both observers. The mean variance in measuring GDAH was $0.23 \mathrm{~mm}$ for observer 1 and $0.07 \mathrm{~mm}$ for observer 2. Standard deviation of the variance for observer 1 was $0.21 \mathrm{~mm}$ and $0.09 \mathrm{~mm}$ for observer 2 . Mean interobserver variability was $0.37 \mathrm{~mm}$.

\section{Results}

Our study reflects a general approach in analyzing GDAH in the cohort of patients and changes in individual patients over time (TP1, TP2, TP3).

In our cohort of 95 patients a total of 170 MRI Scans were performed at different timepoints (TP1, TP2, TP3). Altogether 146 MRIs were collected in ALL and 24 in AML-patients. 49 Patients had only one MRI, 28 patients had two, 7 patients had three and 11 patients had four MRIs (Supplementary Table S1).

The mean GDAH of all MRIs was $33.03 \mathrm{~mm}$ with $32.56 \mathrm{~mm}$ at 
Table 1: GDAH of different cohorts at different timepoints are shown. Significance was tested between all timepoints. $\mathrm{P}<0.05$ is regarded as significant.

\begin{tabular}{|c|c|c|c|c|}
\hline & \multicolumn{3}{|c|}{ Mean ventricular width (mm) } & \multirow{2}{*}{ Significance } \\
\hline & Day 0-30 (TP1) & Day 31-364 (TP2) & Day $\geq 365$ (TP3) & \\
\hline Male & 33,06 & 33,78 & 35,15 & $p=0.006$ \\
\hline Female & 31,97 & 31,51 & 32,89 & \\
\hline Age $\leq 6$ years & 32,36 & 33,09 & 33,99 & $p=0.509$ \\
\hline Age $>6$ years & 32,71 & 32,36 & 34,50 & \\
\hline ALL & 32,40 & 32,75 & 34.31 & $p=0.657$ \\
\hline AML & 33,22 & 32,93 & 36,45 & \\
\hline Patho yes & 32,56 & 32,53 & 34,87 & $p=0.848$ \\
\hline Patho no & 32,55 & 33,36 & 33,79 & \\
\hline High Risk & 31,98 & 32,10 & 35,49 & $p=0.942$ \\
\hline Intermediate Risk & 32,73 & 33,03 & 33,87 & \\
\hline Relapse yes & 33,61 & 33,85 & 35,13 & $p=0.012$ \\
\hline Relapse no & 32,29 & 32,39 & 33,54 & \\
\hline
\end{tabular}

ALL: Acute Lymphoblastic Leukemia; AML: Acute Myeloid Leukemia; Patho: Intracerebral pathomorphological alterations; bold means significant ( $<<0.05$ ); Significant differences in mean GDAH were found between male and female patients $(p=0.006)$ and between patients with relapse and without relapse $(p=0.012)$.

Table 2: The change of GDAH over time is shown. In this analysis, the MRIs of patients with a single MRI are excluded. Significance was tested between all timepoints. $\mathrm{P}<0.05$ is regarded as significant.

\begin{tabular}{|c|c|c|c|c|c|c|}
\hline & \multirow[t]{2}{*}{ Comparisons } & \multirow[t]{2}{*}{$\mathbf{N}$} & \multicolumn{3}{|c|}{$\begin{array}{l}\text { Mean ventricular width } \\
(\mathrm{mm})\end{array}$} & \multirow[t]{2}{*}{ Significance } \\
\hline & & & TP1 & TP2 & TP3 & \\
\hline \multirow{3}{*}{ All } & $\mathrm{TP} 1 \Leftrightarrow \mathrm{TP} 2$ & 24 & 31.7 & 33.0 & & 0.005 \\
\hline & $\mathrm{TP} 1 \Leftrightarrow \mathrm{TP} 3$ & 27 & 33.6 & & 34.3 & 0.08 \\
\hline & $\mathrm{TP} 2 \Leftrightarrow \mathrm{TP} 3$ & 9 & & 33.7 & 32.9 & 0.302 \\
\hline \multirow{3}{*}{ Male } & $\mathrm{TP} 1 \Leftrightarrow \mathrm{TP} 2$ & 13 & 32,0 & 33,4 & & 0.014 \\
\hline & $\mathrm{TP} 1 \Leftrightarrow \mathrm{TP} 3$ & 18 & 34.0 & & 34.6 & 0.18 \\
\hline & $\mathrm{TP} 2 \Leftrightarrow \mathrm{TP} 3$ & 5 & & 34.0 & 33.2 & 0.513 \\
\hline \multirow{3}{*}{ Female } & $\mathrm{TP} 1 \Leftrightarrow \mathrm{TP} 2$ & 11 & 31.2 & 32.5 & & 0.122 \\
\hline & $\mathrm{TP} 1 \Leftrightarrow \mathrm{TP} 3$ & 9 & 32.8 & & 33.5 & 0.269 \\
\hline & $\mathrm{TP} 2 \Leftrightarrow \mathrm{TP} 3$ & 4 & & 33.3 & 32.6 & 0.44 \\
\hline \multirow{3}{*}{ Age $\leq 6$ years } & $\mathrm{TP} 1 \Leftrightarrow \mathrm{TP} 2$ & 9 & 31.5 & 33.3 & & 0.017 \\
\hline & $\mathrm{TP} 1 \Leftrightarrow \mathrm{TP} 3$ & 13 & 33.1 & & 34.1 & 0.028 \\
\hline & $\mathrm{TP} 2 \Leftrightarrow \mathrm{TP} 3$ & 4 & & 33.6 & 32.9 & 0.683 \\
\hline \multirow{3}{*}{ Age $>6$ years } & $\mathrm{TP} 1 \Leftrightarrow \mathrm{TP} 2$ & 15 & 31.7 & 32.7 & & 0.086 \\
\hline & $\mathrm{TP} 1 \Leftrightarrow \mathrm{TP} 3$ & 14 & 34.1 & & 34.4 & 0.624 \\
\hline & $\mathrm{TP} 2 \Leftrightarrow \mathrm{TP} 3$ & 5 & & 33.8 & 33.0 & 0.263 \\
\hline \multirow{3}{*}{ ALL } & $\mathrm{TP} 1 \Leftrightarrow \mathrm{TP} 2$ & 21 & 31.7 & 33.1 & & 0.004 \\
\hline & $\mathrm{TP} 1 \Leftrightarrow \mathrm{TP} 3$ & 25 & 33.5 & & 34.1 & 0.135 \\
\hline & $\mathrm{TP} 2 \Leftrightarrow \mathrm{TP} 3$ & 9 & & 33.7 & 32.9 & 0.302 \\
\hline \multirow{3}{*}{ AML } & $\mathrm{TP} 1 \Leftrightarrow \mathrm{TP} 2$ & 3 & 30.9 & 32.0 & & 0.624 \\
\hline & $\mathrm{TP} 1 \Leftrightarrow \mathrm{TP} 3$ & 2 & 35.1 & & 36.5 & 0.177 \\
\hline & $\mathrm{TP} 2 \Leftrightarrow \mathrm{TP} 3$ & 0 & - & & - & - \\
\hline \multirow{3}{*}{ Patho Yes } & $\mathrm{TP} 1 \Leftrightarrow \mathrm{TP} 2$ & 18 & 31.0 & 32.5 & & 0.004 \\
\hline & $\mathrm{TP} 1 \Leftrightarrow \mathrm{TP} 3$ & 13 & 34.0 & & 34.6 & 0.221 \\
\hline & $\mathrm{TP} 2 \Leftrightarrow \mathrm{TP} 3$ & 8 & & 33.5 & 32.9 & 0.47 \\
\hline
\end{tabular}

\begin{tabular}{|c|c|c|c|c|c|c|}
\hline \multirow{3}{*}{ Patho No } & $\mathrm{TP} 1 \Leftrightarrow \mathrm{TP} 2$ & 6 & 33.6 & 34.3 & & 0.497 \\
\hline & $\mathrm{TP} 1 \Leftrightarrow \mathrm{TP} 3$ & 14 & 33.3 & & 33.9 & 0.231 \\
\hline & $\mathrm{TP} 2 \Leftrightarrow \mathrm{TP} 3$ & 1 & & - & - & - \\
\hline \multirow{3}{*}{ High Risk } & $\mathrm{TP} 1 \Leftrightarrow \mathrm{TP} 2$ & 8 & 30.0 & 31.5 & & 0.123 \\
\hline & $\mathrm{TP} 1 \Leftrightarrow \mathrm{TP} 3$ & 8 & 33.9 & & 35.3 & 0.018 \\
\hline & $\mathrm{TP} 2 \Leftrightarrow \mathrm{TP} 3$ & 2 & & 35.0 & 34.4 & 0.784 \\
\hline \multirow{3}{*}{$\begin{array}{l}\text { Intermediate } \\
\text { Risk }\end{array}$} & $\mathrm{TP} 1 \Leftrightarrow \mathrm{TP} 2$ & 16 & 32.5 & 33.7 & & 0.02 \\
\hline & $\mathrm{TP} 1 \Leftrightarrow \mathrm{TP} 3$ & 19 & 33.5 & & 33.8 & 0.491 \\
\hline & $\mathrm{TP} 2 \Leftrightarrow \mathrm{TP} 3$ & 7 & & 33.3 & 32.5 & 0.364 \\
\hline \multirow{3}{*}{ Relapse Yes } & $\mathrm{TP} 1 \Leftrightarrow \mathrm{TP} 2$ & 6 & 32.3 & 34.2 & & 0.057 \\
\hline & $\mathrm{TP} 1 \Leftrightarrow \mathrm{TP} 3$ & 11 & 34.3 & & 35.5 & 0.017 \\
\hline & $\mathrm{TP} 2 \Leftrightarrow \mathrm{TP} 3$ & 3 & & 34.5 & 34.1 & 0.658 \\
\hline \multirow{3}{*}{ Relapse No } & $\mathrm{TP} 1 \Leftrightarrow \mathrm{TP} 2$ & 18 & 31.4 & 32.6 & & 0.036 \\
\hline & $\mathrm{TP} 1 \Leftrightarrow \mathrm{TP} 3$ & 16 & 33.2 & & 33.4 & 0.673 \\
\hline & $\mathrm{TP} 2 \Leftrightarrow \mathrm{TP} 3$ & 6 & & 33.3 & 32.3 & 0.388 \\
\hline \multirow{3}{*}{ Atrophy Yes } & $\mathrm{TP} 1 \Leftrightarrow \mathrm{TP} 2$ & 2 & 33.8 & 34.7 & & 0.033 \\
\hline & $\mathrm{TP} 1 \Leftrightarrow \mathrm{TP} 3$ & 1 & - & & - & - \\
\hline & TP2 $\Leftrightarrow$ TP3 & 0 & & - & - & - \\
\hline \multirow{3}{*}{ Atrophy No } & $\mathrm{TP} 1 \Leftrightarrow \mathrm{TP} 2$ & 21 & 31.2 & 32.5 & & 0.011 \\
\hline & $\mathrm{TP} 1 \Leftrightarrow \mathrm{TP} 3$ & 24 & 33.1 & & 34.0 & 0.031 \\
\hline & $\mathrm{TP} 2 \Leftrightarrow \mathrm{TP} 3$ & 9 & & 33.7 & 32.9 & 0.302 \\
\hline
\end{tabular}

TP1 = Day 0 - Day 30 after first diagnosis; TP2 = Day 31 - Day 364 after first diagnosis; TP3 = Day $\geq 365$ after first diagnosis; bold means significant $(p<0.05)$; Significant differences in mean GDAH were found in all patients between TP1 and TP2, in male patients between TP1 and TP2, in patients $\leq 6$ years between TP1, and TP2, as well as TP1 and TP3, in ALL patients between TP1 and TP2, in patients with intracerebral pathology between TP1 and TP2, in patients with high risk leukemia between TP1 and TP3, in patients with intermediate risk leukemia between TP1 and TP2, in patients with relapse between TP1 and TP3, in patients without relapse between TP1 and TP2, in patients with morphological atrophy between TP1 and TP2, and in patients without morphological atrophy between TP1 and TP2, as well as TP1 and TP3. 


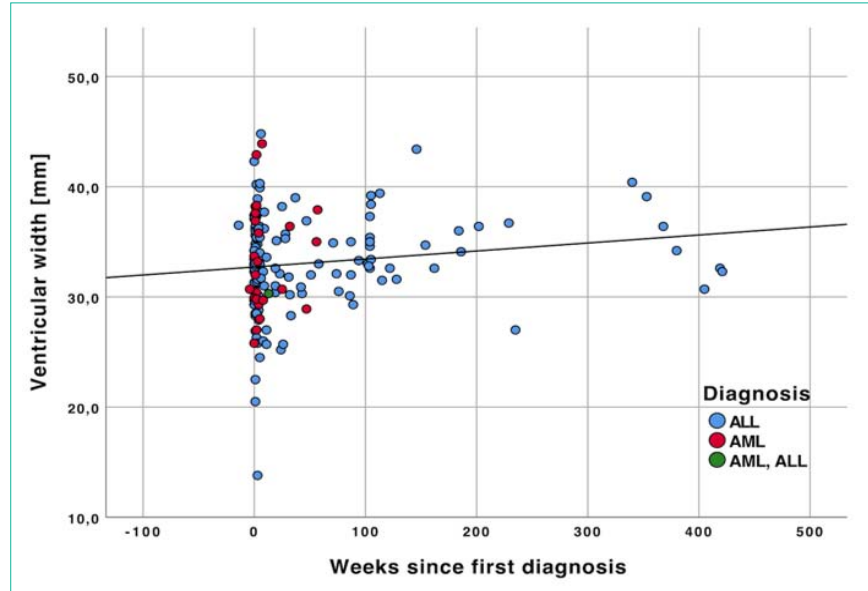

Figure 2: Ventricular width of all MRIs is displayed over time. Measurements for the different diagnoses are shown in different colours.

TP1, 32.62 $\mathrm{mm}$ and $34.58 \mathrm{~mm}$ at TP3.

Patients with ALL showed a significant increase of GDAH in the first year after diagnosis

The mean GDAH of patients with ALL (32.99mm) and AML $(33.42 \mathrm{~mm})$ were not significantly different (Table 1$)$. Nonetheless there was a significant $(\mathrm{p}=0.004)$ increase of GDAH from timepoint TP1 $(31.7 \mathrm{~mm})$ to timepoint TP2 $(33.4 \mathrm{~mm})$ only in the group of patients with ALL. No significant changes between other timepoints were found for ALL and AML (Table 2).

Male patients showed a significant increase of GDAH in the first year after diagnosis

Male patients consistently had significant $(p=0.006)$ larger GDAHs than females at all timepoints. The mean difference between males $(33.82 \mathrm{~mm})$ and females $(32.00 \mathrm{~mm})$ was $1.82 \mathrm{~mm}$.

Regarding individual comparisons between timepoints only boys showed a significant $(\mathrm{p}=0.014)$ increase in GDAH between TP1 and TP2, whereas the increase was not significant in females (Table 2).

\section{Patients up to 6 years of age showed a significant increase of GDAH during follow-up}

In our cohort no significant difference of GDAH between young ( $\leq 6$ years of age) and older children ( $>6$ years of age) could be detected during follow-up time.

However, there was a significant increase of GDAH in individual patients younger than 6 years from TP1 $(31.5 \mathrm{~mm})$ to TP2 $(33.3 \mathrm{~mm})$ $(\mathrm{p}=0.017)$ and from TP1 $(33.1 \mathrm{~mm})$ to TP3 $(34.1 \mathrm{~mm}) \quad(\mathrm{p}=0.028)$ (Table 2).

\section{Patients with pathomorphological changes showed an increase of GDAH in the first year}

Patients without pathomorphological changes showed overall a tendency of increasing GDAH from TP1 (32.6mm) to TP2 (33.4mm) and to TP3 $(33.8 \mathrm{~mm})$ in comparison to patients with additional

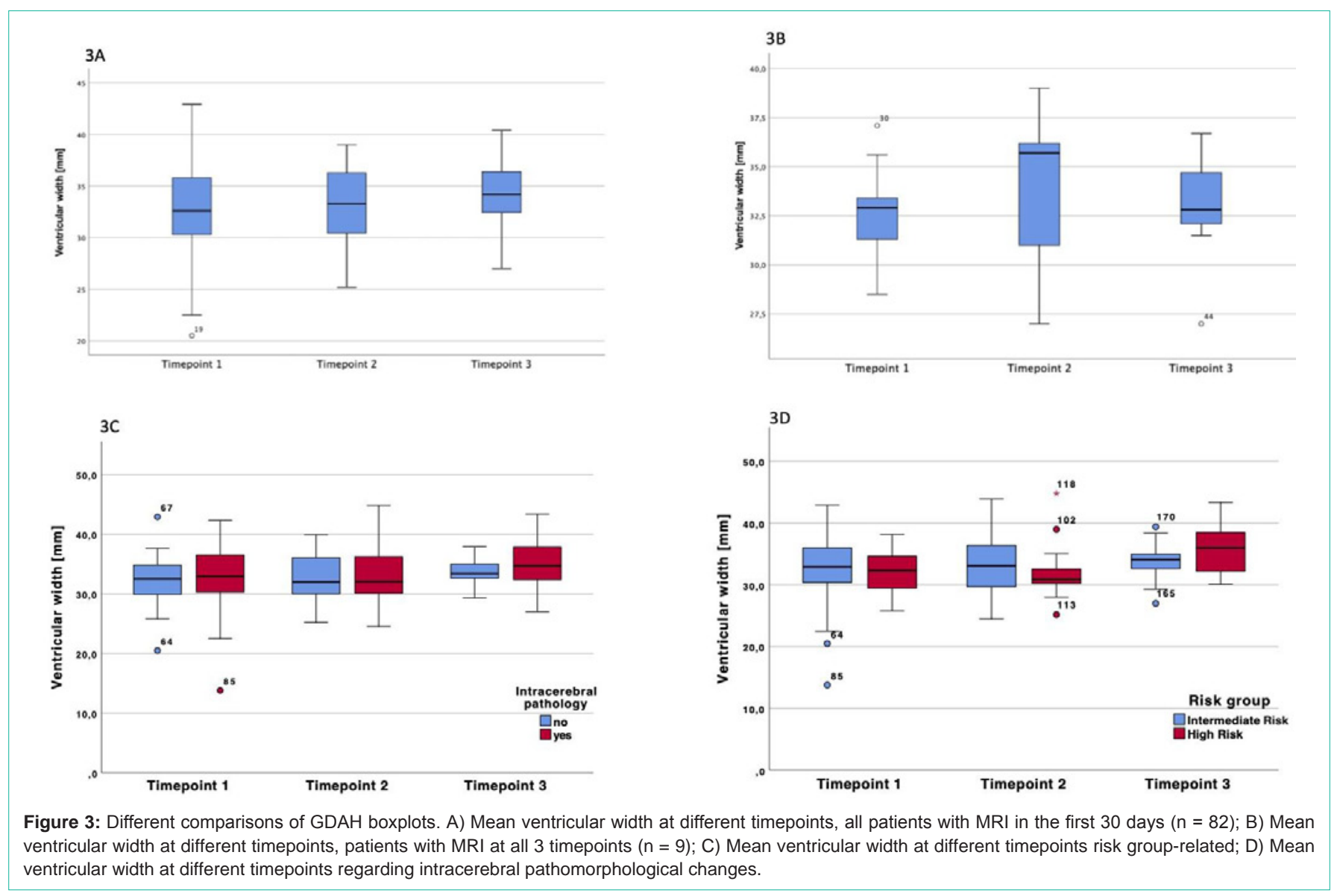


Table 3: Literature overview.

\begin{tabular}{|c|c|c|c|c|c|}
\hline Study & $\mathbf{N}$ & Ventricular width & Findings (Morphology/ Symptoms) & Reason & Recommendations \\
\hline \multicolumn{6}{|c|}{ Long-term studies ( $\geq$ mean 5 years) } \\
\hline $\begin{array}{l}\text { Philips et al., } \\
2019\end{array}$ & 218 & - & $\begin{array}{l}\text { - No significant difference in intracranial } \\
\text { volume between survivors and controls. } \\
\text { Smaller hippocampi and cerebelli in } \\
\text { survivors, thinner cortices in different } \\
\text { regions of the brain (after 5-10 years). }\end{array}$ & Dexamethasone & $\begin{array}{l}\text { - Investigation of benefits } \\
\text { of lower doses of } \\
\text { dexamethasone for } \\
\text { younger female patients. } \\
\text { Early intervention } \\
\text { with NMDA receptor - } \\
\text { antagonist. }\end{array}$ \\
\hline $\begin{array}{l}\text { J.P. } \\
\text { Guenette et } \\
\text { al., } 2016\end{array}$ & $\begin{array}{l}34 \text { (all } \\
\text { CNS- } \\
\text { positive, } \\
\text { mean } 52 \\
\text { years) }\end{array}$ & - & $\begin{array}{l}\text { 74\%: Morphological changes in } \\
\text { cMRI, shorter survival of patients with } \\
\text { pachymeningeal enhancement und at } \\
\text { least two morphological alterations (after } \\
5 \text { years). }\end{array}$ & - & 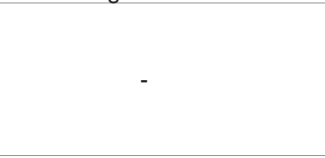 \\
\hline $\begin{array}{l}\text { Badr et al., } \\
\quad 2013\end{array}$ & 25 & - & $\begin{array}{l}\text { 24\%: Alterations, like } \\
\text { leukoencephalopathy (8\%), brain atrophy } \\
(8 \%), \text { old infarcts (4\%), bleedings (4\%); } \\
\text { mainly in high risk -/CNS - /irradiated } \\
\text { patients. } \\
\text { No correlation with age or gender (after } \\
6 \text { years) }\end{array}$ & Cranial irradiation & $\begin{array}{l}\text { - Irradiation should be } \\
\text { indicated restrictively. } \\
\text { MRI scans are a reliable } \\
\text { tool to detect structural } \\
\text { changes of the brain } \\
\text { during therapy. }\end{array}$ \\
\hline $\begin{array}{l}\text { Ficek et al., } \\
2010\end{array}$ & 45 & - & $\begin{array}{l}\text { - } 11 \% \text { : White matter changes, abnormalities } \\
\text { in MRS scans (after 6-12 years) }\end{array}$ & $\begin{array}{l}\text { MTX, Cranial } \\
\text { irradiation }\end{array}$ & $\begin{array}{l}\text { MRS can detect } \\
\text { metabolic effects of } \\
\text { therapy, even if MRI is } \\
\text { still inconspicuous. }\end{array}$ \\
\hline $\begin{array}{l}\text { Porto et al., } \\
2008\end{array}$ & 20 & - & $\begin{array}{l}\text { Decrease of white matter volume (after } \\
\text { mean } 14 \text { years). }\end{array}$ & Cranial irradiation & $\begin{array}{l}\text { Using voxel-based } \\
\text { morphometry to } \\
\text { examine cMRI during } \\
\text { leukemia therapy. } \\
\text { - } \quad \text { Larger cohorts. } \\
\text { Examination of the } \\
\text { connection between } \\
\text { morphology and } \\
\text { neurocognitive abilities. } \\
\text { Examination of } \\
\text { neurogenesis. }\end{array}$ \\
\hline $\begin{array}{l}\text { Dellani et al., } \\
2008\end{array}$ & 13 & - & $\begin{array}{l}2 \text { patients: structural white and gray } \\
\text { matter changes, changes of the } \\
\text { Hippocampus, the Thalami, the temporal } \\
\text { lobe, atrophy (after } 16-28 \text { years) }\end{array}$ & $\begin{array}{l}\text { ALL- Therapy } \\
\text { (mainly cranial } \\
\text { irradiation) }\end{array}$ & $\begin{array}{l}\text { - Follow up- examinations } \\
\text { to detect long-term } \\
\text { sequelae. }\end{array}$ \\
\hline $\begin{array}{l}\text { Pääkkö et } \\
\text { al., } 1994\end{array}$ & $\begin{array}{l}43(27 \\
\text { ALL) }\end{array}$ & $\begin{array}{l}\text { 11/27: Increased ventricular } \\
\text { width }\end{array}$ & $\begin{array}{l}4 \text { patients: white matter changes, reduced } \\
\text { signal (old bleedings) only in irradiated } \\
\text { patients (after 2-20 years) }\end{array}$ & Cranial irradiation & $\begin{array}{l}\text { - If danger of benign brain } \\
\text { tumors: Follow up MRIs } \\
\text { indicated; not necessary } \\
\text { if sole chemotherapy. }\end{array}$ \\
\hline $\begin{array}{l}\text { Lund et al., } \\
1984 \text { (CT- } \\
\text { scans) }\end{array}$ & 28 & $\begin{array}{l}\text { 2/28: Increased ventricular } \\
\text { width }\end{array}$ & $\begin{array}{l}\text { - } 12 \text { patients: slight atrophic changes } \\
\text { - } 9 \text { patients: severe brain atrophy (after } \\
\text { 1-10 years) }\end{array}$ & $\begin{array}{l}\text { Combination } \\
\text { of therapy and } \\
\text { disease severity }\end{array}$ & $\begin{array}{l}\text { Restrictive cranial } \\
\text { irradiation, routine CTs } \\
\text { after diagnosis. }\end{array}$ \\
\hline \multicolumn{6}{|c|}{ Short-term studies (< mean 5 years) } \\
\hline $\begin{array}{l}\text { Morioka et } \\
\text { al., } 2013\end{array}$ & $\begin{array}{l}17(15 \\
\text { ALL })\end{array}$ & - & $\begin{array}{l}\text { DTI scan before and after chemotherapy: } \\
\text { Frontal white matter lesions (after } 3.2 \text { to } \\
6.8 \text { months) }\end{array}$ & $\begin{array}{l}\text { Chemotherapy } \\
\text { (MTX) }\end{array}$ & $\begin{array}{l}\text { Examination of } \\
\text { correlation of } \\
\text { vulnerability by } \\
\text { chemotherapy and } \\
\text { myelination. } \\
\text { - } \quad \text { Long term follow-up. }\end{array}$ \\
\hline $\begin{array}{l}\text { Parasole et } \\
\text { al., } 2010\end{array}$ & $\begin{array}{c}256 \text { ( single } \\
\text { center) }\end{array}$ & - & $\begin{array}{l}27 \text { neurological events (PRES, stroke, } \\
\text { epilepsy, SIADH, MTX- toxicity) (up to } 9 \\
\text { years follow up) }\end{array}$ & $\begin{array}{l}\text { ALL } \\
\text { chemotherapy }\end{array}$ & $\begin{array}{l}\text { Early identification and } \\
\text { fast treatment of CNS } \\
\text { complications to prevent } \\
\text { long-term damage. }\end{array}$ \\
\hline $\begin{array}{l}\text { Porto et al., } \\
2004\end{array}$ & 135 & - & $\begin{array}{l}\text { 22/135 patients: CNS-positive/therapy- } \\
\text { complications (14 ALL, } 7 \text { AML, } 1 \\
\text { AML+ALL). } \\
15 \text { patients before or during therapy: } \\
\text { Sinus thrombosis, cortical vein } \\
\text { thrombosis, bleedings, meningeal } \\
\text { leukemia, infections, skull infiltration of } \\
\text { leukemia. } \\
7 \text { patients after therapy: secondary brain } \\
\text { tumors, skull tumors, microangiopathy, } \\
\text { leukoencephalopathy, white matter } \\
\text { changes, subdural hematomas, irradiation } \\
\text { necrosis, meningeal leukemia. }\end{array}$ & $\begin{array}{l}\text { Chemotherapy, } \\
\text { cranial irradiation }\end{array}$ & $\begin{array}{l}\text { Early diagnosis of CNS- } \\
\text { complications. } \\
\text { Studies to genetic } \\
\text { polymorphism to plan } \\
\text { therapy strategy, } \\
\text { examination of risk } \\
\text { factors. }\end{array}$ \\
\hline
\end{tabular}




\begin{tabular}{|c|c|c|c|c|c|}
\hline $\begin{array}{l}\text { Lo Nigro et } \\
\text { al., } 2000\end{array}$ & 122 & - & $\begin{array}{l}\text { - Acute neurotoxicity in } 3 \text { of } 51 \text { AIEOP-ALL } \\
91 \text { patients and } 7 \text { of } 38 \text { AIEOP-ALL } 95 \text { - } \\
\text { patients }\end{array}$ & $\begin{array}{l}\text { Intrathecal triple } \\
\text { therapy (MTX, } \\
\text { Cytarabine, } \\
\text { Prednisone) }\end{array}$ & - \\
\hline $\begin{array}{l}\text { Mahoney et } \\
\text { al., } 1998\end{array}$ & $\begin{array}{c}1218 \text { (multi } \\
\text { center) }\end{array}$ & - & $\begin{array}{l}95 \text { patients: acute neurotoxicity (mainly } \\
\text { seizures after } 10-11 \text { days), in } 75 \% \text { of } \\
\text { these leukoencephalopathy }\end{array}$ & $\begin{array}{l}\text { MTX (i.v. + i.t.) } \\
\text { triple therapy }\end{array}$ & $\begin{array}{l}\text { Examination of long- } \\
\text { term sequelae. }\end{array}$ \\
\hline $\begin{array}{l}\text { Chen CY et } \\
\text { al., } 1996\end{array}$ & $\begin{array}{c}19(14 \\
\text { ALL, } 4 \\
\text { AML, } 1 \\
\text { AML+ALL) }\end{array}$ & - & $\begin{array}{l}12 \text { patients: Sinus thrombosis / ischemia/ } \\
\text { bleedings/ infections/ meningeal } \\
\text { leukemia/ GBS (All MRIs only if neurologic } \\
\text { symptoms occurred). } \\
\text { - Group 1: Complications during first } 3 \\
\text { months. } \\
\text { - Group 2: Long-term sequelae: } 7 \text { patients: } \\
\text { necrotic leukoencephalopathy (3 years } \\
\text { follow up). }\end{array}$ & $\begin{array}{l}\text { MTX (i.t.), } \\
\text { Cytarabin }\end{array}$ & $\begin{array}{l}\text { Early diagnosis because } \\
\text { a lot of neurologic } \\
\text { complications are } \\
\text { treatable. }\end{array}$ \\
\hline $\begin{array}{l}\text { Pääkkö et } \\
\text { al., } 1996\end{array}$ & $\begin{array}{l}19 \text { (All } \\
\text { CNS } \\
\text { negative, } \\
18 \text { ALL } 1 \\
\text { NHL) }\end{array}$ & $\begin{array}{l}\text { 13/19 pts: Increased } \\
\text { ventricular width (transient) }\end{array}$ & $\begin{array}{l}\text { - } 2 \text { patients: white matter changes (after } \\
\text { 1-29 months). }\end{array}$ & $\begin{array}{l}\text { MTX (i.t.), } \\
\text { increased } \\
\text { ventricular width: } \\
\text { steroids }\end{array}$ & $\begin{array}{l}\text { Therapy related white } \\
\text { matter changes are } \\
\text { rare, follow up MRIs } \\
\text { are not indicated in } \\
\text { asymptomatic patients. }\end{array}$ \\
\hline $\begin{array}{l}\text { Kretzschmar } \\
\text { et al., } 1980 \\
\text { (CT-scans) }\end{array}$ & $\begin{array}{l}72(60 \\
\mathrm{ALL}, 12 \\
\mathrm{NHL})\end{array}$ & $\begin{array}{l}10 \text { pts: Increased ventricular } \\
\text { width before therapy; } \\
8 / 21 \text { pts: Ventricular dilatation } \\
\text { after one year of CNS } \\
\text { treatment }\end{array}$ & $\begin{array}{l}\text { No difference in morphology before and } \\
\text { after therapy (up to } 8 \text { years follow up) }\end{array}$ & $\begin{array}{l}\text { Cranial irradiation, } \\
\text { MTX (i.t.) }\end{array}$ & $\begin{array}{l}\text { Examination, whether } \\
\text { pretherapeutic } \\
\text { ventricular width is a } \\
\text { prognostic factor. }\end{array}$ \\
\hline
\end{tabular}

intracerebral pathomorphologies, where GDAH was significantly $(\mathrm{p}=0.009)$ increasing from TP1 $(32.6 \mathrm{~mm})$ to TP3 $(34.9 \mathrm{~mm})$.

In individual patients, GDAH was significantly $(\mathrm{p}=0.004)$ increasing from TP1 $(31.0 \mathrm{~mm})$ to TP2 $(32.5 \mathrm{~mm})$ in patients with pathomorphological changes (Table 2). Regarding patients without morphological brain atrophy a significant difference in GDAH was seen between TP1 $(31.2 \mathrm{~mm})$ and TP2 $(32.5 \mathrm{~mm})(\mathrm{p}=0.011)$ and between TP1 $(33.1 \mathrm{~mm})$ and TP3 $(34.0 \mathrm{~mm})(\mathrm{p}=0.031)$ in individual patients (Table 2).

\section{Patients with relapse showed an increase of GDAH during follow-up}

Median GDAH in the 19 patients with relapse was significantly $(\mathrm{p}=0.012)$ larger during treatment compared to those patients without relapse $(34.34 \mathrm{~mm}$ vs. $32.51 \mathrm{~mm})$ and their GDAH increased more than for those without relapse $(33.61 \mathrm{~mm}$ (TP1) to $35.13 \mathrm{~mm}$ (TP3) vs. $32.29 \mathrm{~mm}$ (TP1) to $33.54 \mathrm{~mm}$ (TP3)). Furthermore, relapsed patients already had larger ventricles at diagnosis $(33.61 \mathrm{~mm} v s$. $32.29 \mathrm{~mm}$ ) (Table 1).

In individual patients with relapse there was a significant $(\mathrm{p}=0.017)$ increase of GDAH from TP1 $(34.3 \mathrm{~mm})$ to TP3 $(35.5 \mathrm{~mm})$ and non-significant changes $(\mathrm{p}=0.057)$ from TP1 $(32.3 \mathrm{~mm})$ to TP2 $(34.2 \mathrm{~mm})$ and from TP2 $(34.5 \mathrm{~mm})$ to TP3 $(34.1 \mathrm{~mm})$. Non-relapsed patients showed an increase of GDAH from TP1 (31.4mm) to TP2 $(32.6 \mathrm{~mm})$ and a decrease from TP2 $(33.3 \mathrm{~mm})$ to TP3 $(32.3 \mathrm{~mm})$ indicating that ventricular size can also decrease again. Such changes in GDAH over time could only be shown in the individual follow-up of patients (Figure $3 \mathrm{~A}$ and $3 \mathrm{~B}$ and Table 2).

\section{High-risk patients showed a significant increase of GDAH during follow-up}

Comparing high risk with intermediate risk patients, there was an overall difference in the average GDAH of more than $1.6 \mathrm{~mm}$ at TP3 (35.5 to $33.9 \mathrm{~mm}$ ).

On the individual level high-risk (HR) patients developed a significant $(\mathrm{p}=0.018)$ increase of GDAH from TP1 $(33.9 \mathrm{~mm})$ to TP3 $(35.3 \mathrm{~mm})$, Intermediate Risk (IR) patients from TP1 $(32.5 \mathrm{~mm})$ to TP2 only $(33.7 \mathrm{~mm})(\mathrm{p}=0.02)$ (Table 2$)$.

Only in 5 out of 8 patients with cranial irradiation subsequent MRIs were done. Due to the low number, no influence on GDAH was found. The same is true for 5 out of 7 patients with CNS involvement at diagnosis.

\section{Discussion}

It is well known that patients with childhood leukemia can develop neurocognitive sequelae during long term follow-up. This is related to the vulnerable phase during maturation of the brain and neurotoxic treatment elements in prevention or treatment of CNS disease [33-35]. In only few research projects, cranial MRIs were analyzed in childhood leukemia over time to correlate MRI findings with neurocognitive late effects [2-6,36]. Most publications just describe morphological alterations in brain MRIs in this group of patients $[8-22,37]$. We found only 6 articles examining ventricular width in patients with ALL [18-21,36].

In this manuscript, we report our results explicitly analyzing GDAH as a measure of ventricular width in 95 children with leukemia being to the best of our knowledge the largest cohort so far. Our results demonstrate GDAH increases mainly in patients with ALL, recurrence, male gender, high risk, and in patients up to 6 years of age and in those where other pathomorphological findings in cMRI were detected.

Due to the small number of patients with CNS involvement $(n=7)$ and cranial irradiation $(n=8)$, we are unable to answer the question, how intensive CNS treatment in childhood leukemia effects GDAH. Because of the low number of patients with AML, a comparison between the 78 patients with ALL and 17 patients with AML is limited. Only patients with ALL receive dexamethasone, a drug that is known to have neurotoxic effects [22]. This may explain our finding of a significant increase of GDAH in ALL from TP1 to TP2, which is 
not the case in AML. In literature, no study is available addressing the comparison of GDAH between ALL and AML.

Performing literature search in PubMed using the following terms (leukemia (cns late effects or neurotoxicity) (neuroradiology or cns) child $^{*}$ ) and excluding case reports, we detected 16 studies focusing on neuroradiologic late effects in children with leukemia (Table 3).

Kretzschmar et al., 1980 [21] found ventricular dilatation one year after CNS therapy in 8 out of 21 patients and mainly in those with CNS relapse. Whereas in our study increases of GDAH were also found in patients with pathomorphological changes in MRI after one year. Kretzschmar et al. also described a decrease after 2-8 years.

A larger ventricle size was seen in $37 \%$ of patients who received cranial irradiation in the study of Pääkkö et al., 1994 [19]. Because there were only two patients without irradiation, a comparison with irradiated patients was not possible. Furthermore, it is unclear if the ventricular width increased over time or if these patients started already with larger ventricles. In 1996 Pääkkö et al. [18] described a slight to medium dilatation of the sulci and ventricles during ALL therapy.

Hertzberg et al., 1997 [36] detected a ventricular dilatation in $36.8 \%$ of irradiated patients vs. $24.4 \%$ in non-irradiated patients. As prophylactic irradiation is rarely applied today, the number of 8 patients in our cohort is too small to show a generalizable impact on GDAH over time.

The recently published study of Philips et al., 2019 [22] investigated structural MRIs of 218 ALL survivors 5 to 10 years after initial diagnosis. They found that there was no significant difference in intracranial volume between survivors and controls, but former patients with ALL had smaller hippocampi and cerebelli as well as thinner cortices in different regions of the brain, which was correlated to the dose of dexamethasone the patients received. Female patients presented more areas with volume loss than male patients. They found no significant association between MTX and abnormalities of the brain.

Advantages of our single center study are uniform MRI protocols in all our patients and the independent analysis by 2 observers (M.M., W.R.). Nevertheless, a larger cohort with standardized MRI procedures is needed to detect GDAH changes in rare subgroups of childhood leukemia patients, like CNS involvement, cranial irradiation, or AML. We could not find results in literature referring to risk factors like age, gender, risk group, recurrence, or type of leukemia related to an increase in ventricular width. Due to the retrospective nature of this study, a selection bias is possible as cMRIs were only done at diagnosis to rule out CNS involvement or if neurological symptoms occurred. Only those with pathological features did receive cMRIs during follow-up. To overcome this problem routine cMRIs in patients with leukemia at defined timepoints at diagnosis, during treatment and follow-up are necessary to get reliable conclusions of cMRI changes in children with leukemias.

Intrathecal MTX is known for its neurotoxicity [18,38,39]. Further studies with larger cohorts need to be done to look for potential correlation of different doses of intrathecal MTX and the influence on brain. A correlation with specific treatment elements and MRI findings will help to avoid neurotoxic treatment elements if the excellent overall survival can still be guaranteed. We recommend studies correlating morphological findings in MRI with neurocognitive status in children with leukemia. This will allow early interventions in individual patients addressing neurocognitive training. It will be of importance to see how neuroradiological findings will affect neurocognitive sequelae in children with leukemia.

\section{Conclusion}

In summary treatment for childhood, leukemia can increase ventricular width and shows a trend to normalize again in subgroups of patients. We could identify risk factors such as leukemia subtype (ALL), male gender, age up to 6 years, high risk and relapsed patients. Our results show a reliable correlation of childhood leukemia treatment with intracerebral alterations. Based on this study we recommend regular cranial MRI at specific timepoints in children with leukemia to quantify such changes as a basis for correlations with neuropsychological long-term outcome.

\section{Acknowledgement}

Many thanks to PD Dr. Maximilian Linxweiler (Saarland University Medical Center, Department for Ear, Nose and Throat, Homburg, Germany) for proofreading this manuscript and giving valuable recommendations.

\section{References}

1. Rossig C, Juergens $H$, Schrappe $M$, et al. Effective childhood cancer treatment: The impact of large-scale clinical trials in Germany and Austria. Pediatr Blood Cancer. 2013; 60: 1574-1581.

2. Anastasopoulou S, Eriksson MA, Heyman M, et al. Posterior reversible encephalopathy syndrome in children with acute lymphoblastic leukemia: Clinical characteristics, risk factors, course, and outcome of disease. Pediatr Blood Cancer. 2019; 66: e27594.

3. Zając-Spychała O, Pawlak M, Karmelita-Katulska K, et al. Anti-leukemic treatment-induced neurotoxicity in long-term survivors of childhood acute lymphoblastic leukemia: impact of reduced central nervous system radiotherapy and intermediate- to high-dose methotrexate. Leuk Lymphoma. 2018; 59: 2342-2351.

4. Zając-Spychała O, Pawlak MA, Karmelita-Katulska K, Pilarczyk J, Derwich $\mathrm{K}$, Wachowiak J. Long-term brain structural magnetic resonance imaging and cognitive functioning in children treated for acute lymphoblastic leukemia with high-dose methotrexate chemotherapy alone or combined with CNS radiotherapy at reduced total dose to 12 Gy. Neuroradiology. 2017; 59: $147-$ 156.

5. Duffner PK, Armstrong FD, Chen L, et al. Neurocognitive and Neuroradiologic Central Nervous System Late Effects in Children Treated on Pediatric Oncology Group (POG) P9605 (Standard Risk) and P9201 (Lesser Risk) Acute Lymphoblastic Leukemia Protocols (ACCL0131). J Pediatr Hematol Oncol. 2014; 36: 8-15.

6. Steinberg S, Hartmann R, Wisniewski S, Berger K, Beck J, Henze G. Untersuchung von Spätfolgen nach ZNS-Rezidiv einer akuten lymphoblastischen Leukämie im Kindesalter. Klin Pädiatrie. 1998; 210: 200206.

7. Hertzberg H, Huk WJ, Ueberall MA, et al. CNS late effects after ALL therapy in childhood. Part I: Neuroradiological findings in long-term survivors of childhood ALL-an evaluation of the interferences between morphology and neuropsychological performance. Med Pediatr Oncol. 1997; 28: 387-400.

8. Guenette JP, Tirumani $\mathrm{SH}$, Keraliya $A R$, Shinagare $A B$, Ramaiya $N H$, Jagannathan JP. MRI Findings in Patients with Leukemia and Positive CSF Cytology: A Single-Institution 5-Year Experience. Am J Roentgenol. 2016; 207: 1278-1282. 
9. Morioka S, Morimoto M, Yamada K, et al. Effects of chemotherapy on the brain in childhood: diffusion tensor imaging of subtle white matter damage. Neuroradiology. 2013; 55: 1251-1257.

10. Parasole R, Petruzziello F, Menna G, et al. Central nervous system complications during treatment of acute lymphoblastic leukemia in a single pediatric institution. Leuk Lymphoma. 2010; 51: 1063-1071.

11. Ficek K, Blamek S, Syguła D, Miszczyk L, Sońta-Jakimczyk D, Tarnawski R. Evaluation of the late Effects of CNS prophylactic treatment in childhood Acute Lymphoblastic Leukemia (ALL) using magnetic resonance spectroscopy. Acta Neurochirurgica. Supplement. 2010; 106: 195-197.

12. Porto L, Preibisch C, Hattingen E, et al. Voxel-based morphometry and diffusion-tensor MR imaging of the brain in long-term survivors of childhood leukemia. Eur Radiol. 2008; 18: 2691-2700.

13. Dellani PR, Eder S, Gawehn J, et al. Late structural alterations of cerebra white matter in long-term survivors of childhood leukemia. J Magn Reson Imaging. 2008; 27: 1250-1255.

14. Porto L, Kieslich M, Schwabe D, Zanella FE, Lanfermann H. Central nervous system imaging in childhood leukaemia. Eur J Cancer. 2004; 40: 2082-2090.

15. Lo Nigro L, Di Cataldo A, Schiliro G. Acute neurotoxicity in children with B-lineage acute lymphoblastic leukemia (B-ALL) treated with intermediate risk protocols. Med Pediatr Oncol. 2000; 35: 449-455.

16. Mahoney DH, Shuster JJ, Nitschke R, et al. Acute neurotoxicity in children with B-precursor acute lymphoid leukemia: an association with intermediatedose intravenous methotrexate and intrathecal triple therapy-a Pediatric Oncology Group study. J Clin Oncol. 1998; 16: 1712-1722.

17. Cheng-Yu Chen, Robert A Zimmerman, Scott Faro, Larissa T Bilaniuk, Ting-Ywan Chou and PTM. Childhood Leukemia: Central Nervous System Abnormalities during and after Treatment. AJNR Am J Neuroradiol. 1996: 295-310

18. Pääkköö E, Vainionpää L, Pyhtinen J, Lanning M. Minor changes on crania MRI during treatment in children with acute lymphoblastic leukaemia. Neuroradiology. 1996; 38: 264-268.

19. Pääkköö E, Talvensaari K, Pyhtinen J, Lanning M. Late cranial MRI after cranial irradiation in survivors of childhood cancer. Neuroradiology. 1994; 36 : $652-655$

20. Lund E, Hamborg-Pedersen B. Computed tomography of the brain following prophylactic treatment with irradiation therapy and intraspinal methotrexate in children with acute lymphoblastic leukemia. Neuroradiology. 1984; 26: 351 358

21. Kretzschmar K, Gutjahr P, Kutzner J. CT studies before and after CNS treatment for acute lymphoblastic leukemia and malignant non-Hodgkin's lymphoma in childhood. Neuroradiology. 1980; 20: 173-180.

22. Phillips NS, Cheung YT, Glass JO, et al. Neuroanatomical abnormalities related to dexamethasone exposure in survivors of childhood acute lymphoblastic leukemia. Pediatr Blood Cancer. 2020; 67: e27968.

23. Kaatsch P, Grabow D SC. German Childhood Cancer Registry - Annual Report 2017 (1980-2016). Institute of Medical Biostatistics, Epidemiology and Informatics (IMBEI) at the University Medical Center of the Johannes Gutenberg University Mainz. 2018.

24. Hirashima $\mathrm{Y}$, Shindo $\mathrm{K}$, Endo $\mathrm{S}$. Measurement of the area of the anterio horn of the right lateral ventricle for the diagnosis of brain atrophy by CT Correlation with several ventricular indices. Neuroradiology. 1983; 25: 23-27.
25. Pontillo G, Cocozza S, Di Stasi M, et al. 2D linear measures of ventricular enlargement may be relevant markers of brain atrophy and long-term disability progression in multiple sclerosis. Eur Radiol. 2020; 30.

26. Morrow SA, Menon S, Rosehart H, Sharma M. Developing easy to perform routine MRI measurements as potential surrogates for cognitive impairment in MS. Clin Neurol Neurosurg. 2017; 153: 73-78.

27. Ambarki K, Israelsson H, Wåhlin A, Birgander R, Eklund A, Malm J. Brain ventricular size in healthy elderly: Comparison between evans index and volume measurement. Neurosurgery. 2010; 67: 94-99.

28. Wollenweber FA, Schomburg R, Probst M, et al. Width of the third ventricle assessed by transcranial sonography can monitor brain atrophy in a timeand cost-effective manner - Results from a longitudinal study on 500 subjects. Psychiatry Res - Neuroimaging. 2011; 191: 212-216.

29. Greiner J, Schrappe M, Claviez A, et al. THROMBOTECT - a randomized study comparing low molecular weight heparin, antithrombin and unfractionated heparin for thromboprophylaxis during induction therapy of acute lymphoblastic leukemia in children and adolescents. Haematologica. 2019; 104: 756-765.

30. Attarbaschi A, Panzer-Grümayer R, Mann G, et al. Minimal Residual Disease-based Treatment is Adequate for Relapse-prone Childhood Acute Lymphoblastic Leukemia with an intrachromosomal amplification of chromosome 21: The experience of the ALL-BFM 2000 trial. Klin Pädiatrie. 2014; 226: 338-343.

31. Ratei R, Basso G, Dworzak M, et al. Monitoring treatment response of childhood precursor B-cell acute lymphoblastic leukemia in the AIEOP-BFMALL 2000 protocol with multiparameter flow cytometry: predictive impact of early blast reduction on the remission status after induction. Leukemia. 2009; 23: $528-534$.

32. Creutzig $U$, Dworzak M, Zimmermann M, et al. Randomised Introduction of 2-CDA as Intensification during Consolidation for Children with High-risk AML - Results from Study AML-BFM 2004. Klin Pädiatrie. 2015; 227: 116-122.

33. Sleurs C, Lemiere J, Vercruysse T, et al. Intellectual development of childhood ALL patients: a multicenter longitudinal study. Psychooncology. 2017; 26: 508-514.

34. Kanellopoulos A, Andersson S, Zeller B, et al. Neurocognitive outcome in very long-term survivors of childhood acute lymphoblastic leukemia after treatment with chemotherapy only. Pediatr Blood Cancer. 2016; 63: 133-138.

35. Cheung YT, Krull KR. Neurocognitive outcomes in long-term survivors of childhood acute lymphoblastic leukemia treated on contemporary treatment protocols: A systematic review. Neurosci Biobehav Rev. 2015; 53: 108-120.

36. Hertzberg $\mathrm{H}$, Huk WJ, Ueberall MA, et al. CNS late effects after ALL therapy in childhood. Part I: Neuroradiological findings in long-term survivors of childhood ALL-an evaluation of the interferences between morphology and neuropsychological performance. Med Pediatr Oncol. 1997; 28: 387-400.

37. Badr MA, Hassan TH, El-Gerby KM, Lamey ME-S. Magnetic resonance imaging of the brain in survivors of childhood acute lymphoblastic leukemia. Oncol Lett. 2013; 5: 621-626.

38. Yim YS, Mahoney DH, Oshman DG. Hemiparesis and ischemic changes of the white matter after intrathecal therapy for children with acute lymphocytic leukemia. Cancer. 1991; 67: 2058-2061.

39. Salkade P, Lim T. Methotrexate-induced acute toxic leukoencephalopathy. J Cancer Res Ther. 2012; 8: 292. 